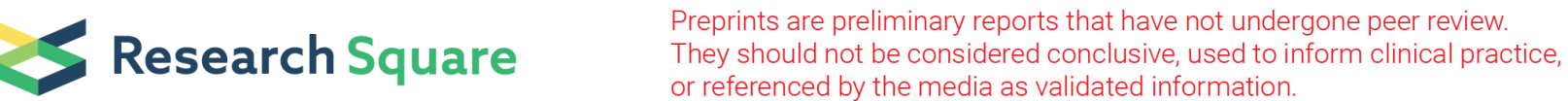

\section{The 5-Factor Modified Frailty Index Predicts Complications and Worsening After Meningioma Surgery in Non-elderly Rather Than Elderly Patients: A Nationwide Registry Study}

Fusao Ikawa ( $\square$ fikawa-nsu@umin.ac.jp )

Graduate School of Biomedical and Helth Sciences, Hiroshima University https://orcid.org/0000-00034205-1490

Nobuaki Michihata

The University of Tokyo

Soichi Oya

Saitama Medical Center

Toshikazu Hidaka

Shimane Prefectural Central Hospital

Shingo Matsuda

Shimane Prefectural Central Hospital

Iori Ozono

Shimane Prefectural Central Hospital

Kenji Ohata

Osaka City University Graduate School of Medicine

Kiyoshi Saito

Fukushima Medical University

Kazunari Yoshida

Keio University School of Medicine

Kiyohide Fushimi

Tokyo Medical and Dental University Graduate School of Medicine

Hideo Yasunaga

The University of Tokyo

Teiji Tominaga

Tohoku University Graduate School of Medicine

Kaoru Kurisu

Hiroshima University 
Keywords: age, complication, five-factor modified frailty index, meningioma, surgery

Posted Date: April 16th, 2021

DOI: https://doi.org/10.21203/rs.3.rs-411629/v1

License: (c) (1) This work is licensed under a Creative Commons Attribution 4.0 International License. Read Full License 


\section{Abstract}

The simplified 5 -factor modified frailty index $(\mathrm{mFI}-5)$ is a useful indicator of outcome for patients undergoing surgeries and considered as an important risk factor in elderly patients. However, its usefulness has not been validated based on age groups. We aimed to investigate the risk factors including the $\mathrm{mFI}-5$ across age groups for complications and worse outcomes in meningioma surgery using data obtained from the nationwide database in Japan. We extracted data from the nationwide registry database in Japan between 2010 and 2015. Age $(<65,65-74$, and $\geq 75$ years), sex, Barthel Index (BI), mFI-5 scores, and complications were evaluated. Multivariate logistic regression analyses identified risk factors across all age groups for worsening $\mathrm{BI}$ scores and complications after surgery. In 8,138 included cases, an mFl-5 score $\geq 2$ items was a significant risk factor for worsening BI scores in patients aged $<65$ years (odds ratio: 2.00; 95\% confidence interval: 1.31-3.06), but not in patients aged 65-74 years and those aged $\geq 75$ years. Similar results were noted for complications in patients aged $<65$ years $(2.40 ; 1.67-3.44)$, but not in patients aged $65-74$ years and those aged $\geq 75$ years. In conclusion, the $\mathrm{mFI}-5$ scores can predict the risk of worsening outcome and complications in non-elderly patients aged $<65$ years rather than in elderly patients aged $\geq 65$ years. In meningioma surgeries, care must be taken when making decisions using the $\mathrm{mFI}-5$ scores based on the patients' age.

Trial Registration

Name: Study on treatment method, age group, complications, and outcome of meningiomas and hemangioblastomas using DPC, URL: http://www.umin.ac.jp/ctr/index-j.htm

ID: UMIN000038486, No.: R000043856

\section{Introduction}

The recent increase in average life expectancy and frequency of diagnostic neuroimaging has resulted in a globally increased rate of incidental meningioma detection in the elderly [14]. Chronological age is considered one of the most effective surgical indications for elderly patients with meningioma worldwide; however, surgical decision-making strategies for elderly patients should be carefully reviewed considering the increased frailty and decline in health that are associated with advanced age.

To assess frailty, the 11-factor modified frailty index (mFl-11) was derived from the Canadian Study of Health and Aging Frailty Index [25] by matching 11 comorbidity and deficit variables from the American College of Surgeons' National Surgery Quality and Improvement Project, which are well-validated health measures that have been applied within general medical and surgical datasets $[7,23,30,33]$. Recently, the simplified 5 -factor modified frailty index $(\mathrm{mFI}-5)$ was introduced and validated within various fields of surgery, and it includes the following five factors: the prevalence of functional dependence, history of diabetes mellitus, history of chronic obstructive pulmonary disease, congestive heart failure, and hypertension $[13,26,28,31]$. Frailty is observed more commonly among the elderly than non-elderly patients; therefore, the frailty index is generally considered an important risk factor in elderly patients. 
However, it remains unknown whether the mFl-5 scores are associated with the same risk across each age group, and whether their predictive value is useful with regard to complications and outcomes of meningioma surgery across various age groups.

Therefore, the aim of this study was to investigate risk factors including the $\mathrm{mFI}-5$ across various age groups for complications and worse outcomes in meningioma surgery, with data extracted from the nationwide database in Japan.

\section{Methods}

\section{Protocol approval and patient consent}

The present study was approved by the local Institutional Review Boards of Hiroshima University (no. E631) and Tokyo University (no. 3501-[1]). Due to the anonymous nature of the data in this study, the requirement for informed consent was waived.

\section{Data source and selection of patients}

The Japanese Diagnosis Procedure Combination (DPC) is a registry-based national database that includes abstract discharge data and administrative claims on inpatients in Japan. It has been described thoroughly elsewhere $[10,11,17]$. Both the sensitivity and specificity of the procedure exceeded $90 \%$ [32].

We included patients aged 18-95 years who were admitted to the hospital with a primary diagnosis of intracranial meningioma between July 1, 2010, and March 31, 2015. Diagnoses of meningioma (ICD-10 codes; D32) and the intracranial tumor removal procedure (medical fee code; K169) were identified. In total, 10,530 patients with meningiomas were identified, and we excluded cases with unknown location of the meningioma, multiple meningiomas, and no detection of $\mathrm{BI}$ assessments and body mass index (BMI). Consequently, 8,138 patients with meningiomas were included in this study (Fig. 1).

The database incorporates the coded variables as per the International Classification of Diseases and Related Health Problems, 10th Revision (ICD-10), and the Barthel Index (BI) at admission and at discharge were evaluated. We collected data on the patients' sex, age, BMI, medical history, internal oral medication on admission, location of the meningioma, BI score at admission and at discharge, and in-hospital complications. As part of the medical history, we included a review of diabetes mellitus (ICD-10 code: E14), hypertension (I10), cerebral infarction (I63), angina pectoris (I20), congestive heart failure (I50), and chronic obstructive pulmonary disease (J44). We reviewed the use of antiplatelet drugs (aspirin, ticlopidine hydrochloride, cilostazol, clopidogrel sulfate, clopidogrel-aspirin combination, and prasugrel hydrochloride), anticoagulation agents (warfarin, dabigatran etexilate, edoxaban tosilate hydrate, rivaroxaban, and apixaban), and statins (atorvastatin calcium hydrate, rosuvastatin calcium, and pitavastatin calcium hydrate). In addition, the patients for the presence of complications, including intracerebral hemorrhage (ICH; ICD-10 code: I61), subarachnoid hemorrhage (SAH; I60), cerebral infarction, congestive heart failure, and pneumonia (J18) was evaluated. 
The $\mathrm{BI}$ was used to evaluate 10 activities of daily living (ADL) across two to four stages [2, 29]. The BI was classified into three categories: $0-55,60-80$, and 85-100 points according to ADL, with higher scores indicating a higher level of independent functioning. One of the five factors included in the $\mathrm{mFI}-5$ was the functional status of requiring assistance with ADL. We defined this dependent functional status as a $\mathrm{BI}$ score $<85$. The scores of the $\mathrm{mFI}-5$ were classified into three categories, namely, 0,1 item, and $\geq 2$ items. A worsening $\mathrm{BI}$ score indicated patients who demonstrated a decreased $\mathrm{BI}$ score $\geq 5$ points at discharge compared to that at admission, and in-hospital mortality indicated death from any cause. Any complications included any stroke, congestive heart failure, pneumonia, a decreased BI score, and inhospital mortality.

The hospital data reviewed primarily assessed the case volume and type (academic or non-academic). Hospital case volume was defined as the number of patients with meningiomas treated surgically at an individual facility during the study period, and this was categorized into three groups according to terciles of case volume, with an approximately equal number of patients in each of the three groups. Hospital case volume was categorized from 1 to 3 , ordered from the lowest to the highest value. The anatomical locations of the meningiomas were classified as convexity, falx, parasagittal, lateral (sphenoidal ridge, middle fossa), midline (tuberculum sellae, olfactory groove), posterior fossa (foramen magnum, petrous, petroclival, petrotentorial, tentorial), or deep (ventricle, anterior clinoid, posterior clinoid, cavernous, orbital, falcotentorial). Patients were categorized into three age groups, based on the classification of the World Health Organization and the Japan Geriatrics Society, as follows: < 65 years (non-elderly), 65-74 years (pre-elderly), and $\geq 75$ years (elderly).[18] All patients according to their BMI were classified into the following groups: $<18.5 \mathrm{~kg} / \mathrm{m}^{2}, 18.5-24.9 \mathrm{~kg} / \mathrm{m}^{2}$ (healthy weight), $25.0-29.9 \mathrm{~kg} / \mathrm{m}^{2}$, and $\geq 30.0 \mathrm{~kg} / \mathrm{m}^{2}$.

\section{Statistical analyses}

All statistical analyses were performed using Stata (version 15; StataCorp, College Station, TX, USA). Categorical variables were compared using a Chi-square or Fisher's exact test. To compare continuous variables, we performed a t-test or Mann-Whitney $U$ test. Multivariate logistic regression analyses were performed on the overall cohort to analyze the risk factors for worsening $\mathrm{BI}$ scores between admission and discharge, in-hospital mortality, and any complications. The same analyses were performed separately across the three categories of age groups, except for in-hospital mortality where the numbers of participants in each subgroup were too low for statistical analysis. For multivariate logistic regression analyses, independent variables were selected based on the existing literature $[4,13,19,20,26,28,31]$, and no variable selection method was applied; odds ratios (ORs) and 95\% confidence intervals (Cls) were calculated.

\section{Results}

During the study period, 8,138 eligible patients were surgically treated. The mean (interquartile range) age was 63.0 (range: 53.0-71.0). Table 1 shows the baseline characteristics of patients with surgically treated meningioma based on age group. The mFI-5 score was significantly increased with age. Elderly 
patients tended to undergo surgery in lower surgical volume and non-academic hospitals. The prevalence of ICH, SAH, cerebral infarction, congestive heart failure, and in-hospital mortality did not significantly increase with age. However, the prevalence of pneumonia, worsening BI scores, and any complications increased significantly with age. 
Table 1

Baseline characteristics of patients surgically treated for meningioma based on age groups

\begin{tabular}{|c|c|c|c|c|}
\hline Year age group (years) & $\begin{array}{l}\text { Non-elderly; }< \\
65\end{array}$ & $\begin{array}{l}\text { Preelderly; 65- } \\
74\end{array}$ & Elderly; $\geq 75$ & \\
\hline No. & 4,378 & 2,421 & 1,339 & $P$-value \\
\hline Sex (male) & 1305 (29.8\%) & $760(31.4 \%)$ & $479(35.8 \%)$ & $<.001 *$ \\
\hline Age, median (IQR) & $\begin{array}{l}54.0(46.0 \\
60.0)\end{array}$ & $\begin{array}{l}69.0(67.0 \\
72.0)\end{array}$ & $\begin{array}{l}78.0(76.0 \\
81.0)\end{array}$ & <. \\
\hline BMI, median (IQR) & $\begin{array}{l}22.7(20.4 \\
25.4)\end{array}$ & $\begin{array}{l}23.0(20.8 \\
25.3)\end{array}$ & $\begin{array}{l}22.9(20.8 \\
25.4)\end{array}$ & 0.082 \\
\hline BMI classification (kg/m2) & & & & $\dot{0.001 *}$ \\
\hline$<18.5$ & $351(8.0 \%)$ & $167(6.9 \%)$ & $116(8.7 \%)$ & \\
\hline $18.5-24.9$ & $2,821(64.4 \%)$ & $1,578(65.2 \%)$ & $851(63.6 \%)$ & \\
\hline $25-29.9$ & $942(21.5 \%)$ & $574(23.7 \%)$ & $321(24.0 \%)$ & \\
\hline $30 \leq$ & $264(6.0 \%)$ & $102(4.2 \%)$ & $51(3.8 \%)$ & \\
\hline \multicolumn{5}{|l|}{ Medical history } \\
\hline Diabetes mellitus & $387(8.8 \%)$ & $395(16.3 \%)$ & $218(16.3 \%)$ & $<.001 *$ \\
\hline Hypertension & $736(16.8 \%)$ & $789(32.6 \%)$ & $494(36.9 \%)$ & $<.001^{*}$ \\
\hline Cerebral infarction & $35(0.8 \%)$ & $48(2.0 \%)$ & $34(2.5 \%)$ & $<.001 *$ \\
\hline Angina pectoris & $51(1.2 \%)$ & $69(2.9 \%)$ & $68(5.1 \%)$ & <. $001 *$ \\
\hline Congestive heart failure & $20(0.5 \%)$ & $26(1.1 \%)$ & $36(2.7 \%)$ & $<.001 *$ \\
\hline $\begin{array}{l}\text { Chronic obstructive pulmonary } \\
\text { disease }\end{array}$ & $1(0.02 \%)$ & $8(0.3 \%)$ & $4(0.3 \%)$ & $0.004^{*}$ \\
\hline The modified frailty index-5 & & & & $<.001 *$ \\
\hline 0 item & $3112(71.1 \%)$ & $1202(49.6 \%)$ & $475(35.5 \%)$ & \\
\hline
\end{tabular}

$\mathrm{BI}=$ Barthel Index; $\mathrm{BMI}=$ body mass index; $\mathrm{ICH}$ = intracerebral hemorrhage; IQR = interquartile range; No. = number; $\mathrm{SAH}=$ subarachnoid hemorrhage;

* $p<0.05$ 


\begin{tabular}{|c|c|c|c|c|}
\hline Year age group (years) & $\begin{array}{l}\text { Non-elderly; < } \\
65\end{array}$ & $\begin{array}{l}\text { Preelderly; } 65- \\
74\end{array}$ & Elderly; $\geq 75$ & \\
\hline 1 item & $1023(23.4 \%)$ & $910(37.6 \%)$ & $583(43.5 \%)$ & \\
\hline$\geq 2$ items & $243(5.6 \%)$ & $309(12.8 \%)$ & $281(21.0 \%)$ & \\
\hline \multicolumn{5}{|l|}{$\begin{array}{l}\text { Internal oral medication on } \\
\text { admission }\end{array}$} \\
\hline Antiplatelet & $84(1.9 \%)$ & $139(5.7 \%)$ & $126(9.4 \%)$ & $\begin{array}{l}< \\
0.001 *\end{array}$ \\
\hline Anticoagulation & $72(1.6 \%)$ & $105(4.3 \%)$ & $107(8.0 \%)$ & $\stackrel{<}{0.001 *}$ \\
\hline Statin & $253(5.8 \%)$ & $279(11.5 \%)$ & $178(13.3 \%)$ & $\begin{array}{l}<.001 * \\
0.0\end{array}$ \\
\hline $\begin{array}{l}\text { Barthel index on admission } \\
\text { (points) }\end{array}$ & & & & $<.001 *$ \\
\hline $0-55$ & $264(6.0 \%)$ & $234(9.7 \%)$ & $314(23.5 \%)$ & \\
\hline $60-80$ & $119(2.7 \%)$ & $117(4.8 \%)$ & $113(8.4 \%)$ & \\
\hline $85-100$ & 3,995 (91.3\%) & $2,070(85.5 \%)$ & $912(68.1 \%)$ & \\
\hline Location & & & & $<.001 *$ \\
\hline Convexity & $1,414(32.3 \%)$ & $809(33.4 \%)$ & $565(42.2 \%)$ & \\
\hline Falx & 469 (10.7\%) & $291(12.0 \%)$ & $136(10.2 \%)$ & \\
\hline Parasagittal & 741 (16.9\%) & 351 (14.5\%) & $141(10.5 \%)$ & \\
\hline Lateral & $491(11.2 \%)$ & $277(11.4 \%)$ & $166(12.4 \%)$ & \\
\hline Midline & $473(10.8 \%)$ & $274(11.3 \%)$ & $84(6.3 \%)$ & \\
\hline Posterior fossa & $672(15.3 \%)$ & 381 (15.7\%) & $233(17.4 \%)$ & \\
\hline Deep & $118(2.7 \%)$ & $38(1.6 \%)$ & $14(1.0 \%)$ & \\
\hline Hospital volume & & & & $<.001^{*}$ \\
\hline 1 & $1,296(29.6 \%)$ & $866(35.8 \%)$ & $516(38.5 \%)$ & \\
\hline 2 & $1,402(32.0 \%)$ & $765(31.6 \%)$ & 449 (33.5\%) & \\
\hline
\end{tabular}

$\mathrm{BI}=\mathrm{Barthel}$ Index; $\mathrm{BMI}=$ body mass index; $\mathrm{ICH}$ = intracerebral hemorrhage; IQR = interquartile range; No. = number; $\mathrm{SAH}=$ subarachnoid hemorrhage;

* $p<0.05$ 


\begin{tabular}{|c|c|c|c|c|}
\hline Year age group (years) & $\begin{array}{l}\text { Non-elderly; }< \\
65\end{array}$ & $\begin{array}{l}\text { Preelderly; 65- } \\
74\end{array}$ & Elderly; $\geq 75$ & \\
\hline 3 & $1,680(38.4 \%)$ & $790(32.6 \%)$ & $374(27.9 \%)$ & \\
\hline Academic & $1,838(42.0 \%)$ & $861(35.6 \%)$ & $422(31.5 \%)$ & 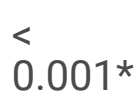 \\
\hline Barthel index at discharge (points) & & & & 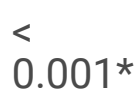 \\
\hline $0-55$ & $141(3.2 \%)$ & $194(8.1 \%)$ & $278(20.9 \%)$ & \\
\hline $60-80$ & $137(3.1 \%)$ & $126(5.2 \%)$ & $161(12.1 \%)$ & \\
\hline $85-100$ & $4,079(93.6 \%)$ & $2,089(86.7 \%)$ & $888(66.9 \%)$ & \\
\hline \multicolumn{5}{|l|}{ Complication } \\
\hline $\mathrm{ICH}$ & $16(0.4 \%)$ & $14(0.6 \%)$ & $9(0.7 \%)$ & 0.260 \\
\hline SAH & $3(0.1 \%)$ & $1(<1 \%)$ & $0(0.0 \%)$ & 0.600 \\
\hline Cerebral infarction & $85(1.9 \%)$ & $68(2.8 \%)$ & $30(2.2 \%)$ & 0.069 \\
\hline Congestive heart failure & $15(0.3 \%)$ & $13(0.5 \%)$ & $8(0.6 \%)$ & 0.330 \\
\hline Pneumonia & $81(1.9 \%)$ & $73(3.0 \%)$ & $56(4.2 \%)$ & $<.001 *$ \\
\hline Worsening BI scores & $311(7.1 \%)$ & $305(12.6 \%)$ & $283(21.1 \%)$ & 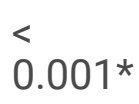 \\
\hline In-hospital mortality & $22(0.5 \%)$ & $12(0.5 \%)$ & $12(0.9 \%)$ & 0.210 \\
\hline Any complications & $454(10.4 \%)$ & $422(17.4 \%)$ & $341(25.5 \%)$ & $<.001 *$ \\
\hline \multicolumn{5}{|c|}{$\begin{array}{l}\mathrm{BI}=\text { Barthel Index; } \mathrm{BMI}=\text { body mass index; ICH = intracerebral hemorrhage; IQR = interquartile range; } \\
\text { No. = number; } \mathrm{SAH}=\text { subarachnoid hemorrhage; }\end{array}$} \\
\hline$* p<0.05$ & & & & \\
\hline
\end{tabular}

Table 2 depicts the results of the multivariate logistic regression analyses for worsening BI scores, inhospital mortality, and any complications in all the cases. For worsening BI scores, significant risk factors included being in the pre-elderly group (OR: $1.77 ; 95 \% \mathrm{Cl}: 1.49-2.11)$ and in the elderly group $(3.21 ; 2.65-$ $3.88)$, cerebral infarction (1.65; 1.03-2.65), an mFI-5 score $\geq 2$ items $(1.29 ; 1.03-1.62)$, anticoagulants on admission, and meningioma location (parasagittal, lateral, and deep). For in-hospital mortality, BMI < 18.5 $\mathrm{kg} / \mathrm{m}^{2}$ (3.30; 1.44-7.57), an mFI-5 score $\geq 2$ items (5.37; 2.34-12.30), antiplatelet oral medications on admission, and tumor location (falx, parasagittal, midline, and posterior fossa) were significant risk factors, whereas hospital volume $3(0.33 ; 0.14-0.82)$ was a significant inverse risk factor. For any complications, significant risk factors included being in the pre-elderly group (OR: $1.67 ; 95 \% \mathrm{Cl}$ : $1.44-$ $1.93)$ and in the elderly group $(2.55 ; 2.15-3.02), \mathrm{BMI}<18.5$ (1.26; 1.00-1.58), an mFI-5 score $\geq 2$ items 
$(1.54 ; 1.26-1.88)$, oral medications on the admission of antiplatelets and anticoagulants, and tumor location (falx, parasagittal, midline, posterior fossa, and deep). Figure 2 ( $a, b$, and c) depicts, in the form of forest plots for risk factors adjusted for other variables, the results of the multivariate logistic regression analysis for worsening BI scores, in-hospital mortality, and any complications in the cases of all ages. 
Table 2

Multivariate logistic regression analyses for worsening BI scores, in-hospital mortality, and any complications in the total cases

\begin{tabular}{|c|c|c|c|c|c|c|}
\hline \multirow[t]{2}{*}{ Objective variables (No.) } & \multicolumn{2}{|c|}{$\begin{array}{l}\text { Worsening BI scores } \\
(8,138)\end{array}$} & \multicolumn{2}{|c|}{$\begin{array}{l}\text { In-hospital mortality } \\
(7,428)\end{array}$} & \multicolumn{2}{|c|}{$\begin{array}{l}\text { Any complications } \\
(8,138)\end{array}$} \\
\hline & $\begin{array}{l}\mathrm{OR}(95 \% \\
\mathrm{Cl})\end{array}$ & $\begin{array}{l}P \\
\text { value }\end{array}$ & OR (95\% Cl) & $\begin{array}{l}P \\
\text { value }\end{array}$ & $\begin{array}{l}\mathrm{OR}(95 \% \\
\mathrm{Cl})\end{array}$ & $\begin{array}{l}P \\
\text { value }\end{array}$ \\
\hline Sex male & $\begin{array}{l}1.04(0.89- \\
1.21)\end{array}$ & 0.655 & $\begin{array}{l}1.41(0.76- \\
2.61)\end{array}$ & 0.273 & $\begin{array}{l}1.07 \\
(0.93- \\
1.22)\end{array}$ & 0.356 \\
\hline \multicolumn{7}{|l|}{ Age group (years) } \\
\hline Non-elderly; $<65$ & Reference & & Reference & & Reference & \\
\hline Pre-elderly; 65-74 & $\begin{array}{l}1.77(1.49- \\
2.11)\end{array}$ & $\begin{array}{l}<.001 * \\
\end{array}$ & $\begin{array}{l}0.60(0.28- \\
1.29)\end{array}$ & 0.192 & $\begin{array}{l}1.67 \\
(1.44- \\
1.93)\end{array}$ & $<.001 *$ \\
\hline Elderly; $\geq 75$ & $\begin{array}{l}3.21(2.65- \\
3.88)\end{array}$ & $\dot{0.001 *}$ & $\begin{array}{l}1.09(0.50- \\
2.38)\end{array}$ & 0.829 & $\begin{array}{l}2.55 \\
(2.15- \\
3.02)\end{array}$ & $<.001 *$ \\
\hline \multicolumn{7}{|l|}{$\begin{array}{l}\text { BMI classification } \\
(\mathrm{kg} / \mathrm{m} 2)\end{array}$} \\
\hline$<18.5$ & $\begin{array}{l}1.15(0.89- \\
1.49)\end{array}$ & 0.285 & $\begin{array}{l}3.30(1.44- \\
7.57)\end{array}$ & $0.005^{\star}$ & $\begin{array}{l}1.26(1.00- \\
1.58)\end{array}$ & $0.050 *$ \\
\hline $18.5-24.9$ & Reference & & Reference & & Reference & \\
\hline $25-29.9$ & $\begin{array}{l}1.03(0.86- \\
1.22)\end{array}$ & 0.774 & $\begin{array}{l}1.29(0.62- \\
2.69)\end{array}$ & 0.493 & $\begin{array}{l}1.04 \\
(0.90- \\
1.22)\end{array}$ & 0.575 \\
\hline $30 \leq$ & $\begin{array}{l}0.85(0.60- \\
1.21)\end{array}$ & 0.370 & $\begin{array}{l}1.60(0.53- \\
4.85)\end{array}$ & 0.404 & $\begin{array}{l}0.89 \\
(0.66- \\
1.20)\end{array}$ & 0.441 \\
\hline \multicolumn{7}{|l|}{ Medical history } \\
\hline Cerebral infarction & $\begin{array}{l}1.65(1.03- \\
2.65)\end{array}$ & $0.035^{\star}$ & $\begin{array}{l}3.31(0.94- \\
11.68)\end{array}$ & 0.063 & $\begin{array}{l}1.27 \\
(0.82- \\
1.98)\end{array}$ & 0.289 \\
\hline Angina pectoris & $\begin{array}{l}1.02(0.67- \\
1.54)\end{array}$ & 0.936 & $\begin{array}{l}0.63(0.08- \\
4.97)\end{array}$ & 0.664 & $\begin{array}{l}0.81 \\
(0.55- \\
1.19)\end{array}$ & 0.284 \\
\hline \multicolumn{7}{|l|}{$\begin{array}{l}\text { The modified frailty index- } \\
5\end{array}$} \\
\hline \multicolumn{7}{|c|}{$\mathrm{BI}=$ Barthel Index; $\mathrm{BMI}=$ body mass index; $\mathrm{Cl}=$ confidence interval; $\mathrm{OR}$ = odds ratio; } \\
\hline$* p<0.05$ & & & & & & \\
\hline
\end{tabular}




\begin{tabular}{|c|c|c|c|c|c|c|}
\hline \multirow[t]{2}{*}{ Objective variables (No.) } & \multicolumn{2}{|c|}{$\begin{array}{l}\text { Worsening BI scores } \\
(8,138)\end{array}$} & \multicolumn{2}{|c|}{$\begin{array}{l}\text { In-hospital mortality } \\
(7,428)\end{array}$} & \multicolumn{2}{|c|}{$\begin{array}{l}\text { Any complications } \\
(8,138)\end{array}$} \\
\hline & $\begin{array}{l}\mathrm{OR}(95 \% \\
\mathrm{Cl})\end{array}$ & $\begin{array}{l}P \\
\text { value }\end{array}$ & OR $(95 \% \mathrm{Cl})$ & $\begin{array}{l}P \\
\text { value }\end{array}$ & $\begin{array}{l}\mathrm{OR}(95 \% \\
\mathrm{Cl})\end{array}$ & $\begin{array}{l}P \\
\text { value }\end{array}$ \\
\hline 0 item & Reference & & Reference & & Reference & \\
\hline 1 item & $\begin{array}{l}1.08(0.92- \\
1.28)\end{array}$ & 0.357 & $\begin{array}{l}2.53(1.22- \\
5.22)\end{array}$ & $0.012^{*}$ & $\begin{array}{l}1.14 \\
(0.99- \\
1.32)\end{array}$ & 0.074 \\
\hline$\geq 2$ items & $\begin{array}{l}1.29(1.03- \\
1.62)\end{array}$ & $0.026^{*}$ & $\begin{array}{l}5.37(2.34- \\
12.30)\end{array}$ & $<.001 *$ & $\begin{array}{l}1.54 \\
(1.26- \\
1.88)\end{array}$ & $\begin{array}{l}<.001 * \\
0.00\end{array}$ \\
\hline \multicolumn{7}{|l|}{$\begin{array}{l}\text { Internal oral medication } \\
\text { on admission }\end{array}$} \\
\hline Antiplatelet & $\begin{array}{l}1.26(0.93- \\
1.71)\end{array}$ & 0.128 & $\begin{array}{l}3.63(1.49- \\
8.85)\end{array}$ & $0.005^{\star}$ & $\begin{array}{l}2.00 \\
(1.54- \\
2.58)\end{array}$ & $<.001 *$ \\
\hline Anticoagulation & $\begin{array}{l}2.28(1.71- \\
3.03)\end{array}$ & <. $001 *$ & $\begin{array}{l}1.66(0.56- \\
4.90)\end{array}$ & 0.357 & $\begin{array}{l}2.56 \\
(1.97- \\
3.32)\end{array}$ & $\begin{array}{l}<.001 * \\
0.0\end{array}$ \\
\hline Statin & $\begin{array}{l}1.19(0.94- \\
1.49)\end{array}$ & 0.150 & $\mathrm{~N} / \mathrm{A}$ & & $\begin{array}{l}1.03 \\
(0.83- \\
1.27)\end{array}$ & 0.796 \\
\hline \multicolumn{7}{|l|}{ Location } \\
\hline Convexity & Reference & & Reference & & Reference & \\
\hline Falx & $\begin{array}{l}1.45(1.13- \\
1.84)\end{array}$ & $0.003^{\star}$ & $\begin{array}{l}4.84(1.51- \\
15.55)\end{array}$ & $0.008^{*}$ & $\begin{array}{l}1.38 \\
(1.12- \\
1.71)\end{array}$ & $0.003^{*}$ \\
\hline Parasagittal & $\begin{array}{l}1.87(1.51- \\
2.32)\end{array}$ & $\dot{0} .001 *$ & $\begin{array}{l}5.17(1.64- \\
16.34)\end{array}$ & $0.005^{\star}$ & $\begin{array}{l}1.73 \\
(1.43- \\
2.09)\end{array}$ & $<.001 *$ \\
\hline Lateral & $\begin{array}{l}1.33(1.05- \\
1.70)\end{array}$ & $0.021^{\star}$ & $\begin{array}{l}0.64(0.07- \\
5.49)\end{array}$ & 0.681 & $\begin{array}{l}1.02 \\
(0.82- \\
1.28)\end{array}$ & 0.839 \\
\hline Midline & $\begin{array}{l}1.17(0.89- \\
1.53)\end{array}$ & 0.263 & $\begin{array}{l}9.27(3.06- \\
28.13)\end{array}$ & $<.001 *$ & $\begin{array}{l}1.26(1.00- \\
1.58)\end{array}$ & $0.049 *$ \\
\hline Posterior fossa & $\begin{array}{l}1.22(0.98- \\
1.53)\end{array}$ & 0.076 & $\begin{array}{l}6.96(2.44- \\
19.81)\end{array}$ & $\begin{array}{l}<.001 * \\
0.00\end{array}$ & $\begin{array}{l}1.29 \\
(1.06- \\
1.56)\end{array}$ & $0.010 *$ \\
\hline \multicolumn{7}{|c|}{$\mathrm{BI}=\mathrm{Barthel}$ Index; $\mathrm{BMI}=$ body mass index; $\mathrm{Cl}=$ confidence interval; $\mathrm{OR}=$ odds ratio; } \\
\hline$* p<0.05$ & & & & & & \\
\hline
\end{tabular}




\begin{tabular}{|c|c|c|c|c|c|c|}
\hline \multirow[t]{2}{*}{ Objective variables (No.) } & \multicolumn{2}{|c|}{$\begin{array}{l}\text { Worsening Bl scores } \\
(8,138)\end{array}$} & \multicolumn{2}{|c|}{$\begin{array}{l}\text { In-hospital mortality } \\
(7,428)\end{array}$} & \multicolumn{2}{|c|}{$\begin{array}{l}\text { Any complications } \\
(8,138)\end{array}$} \\
\hline & $\begin{array}{l}\text { OR }(95 \% \\
\mathrm{Cl})\end{array}$ & $\begin{array}{l}P \\
\text { value }\end{array}$ & OR $(95 \% \mathrm{Cl})$ & $\begin{array}{l}P \\
\text { value }\end{array}$ & $\begin{array}{l}\text { OR }(95 \% \\
\mathrm{Cl})\end{array}$ & $\begin{array}{l}P \\
\text { value }\end{array}$ \\
\hline Deep & $\begin{array}{l}2.55(1.64- \\
3.94)\end{array}$ & $<.001 *$ & $\begin{array}{l}9.97(1.85- \\
53.85)\end{array}$ & $0.008^{*}$ & $\begin{array}{l}2.14 \\
(1.43- \\
3.20)\end{array}$ & $<.001^{*}$ \\
\hline \multicolumn{7}{|l|}{ Hospital volume } \\
\hline 1 & \multicolumn{2}{|l|}{ Reference } & \multicolumn{2}{|l|}{ Reference } & \multicolumn{2}{|l|}{ Reference } \\
\hline 2 & $\begin{array}{l}0.97(0.81- \\
1.16)\end{array}$ & 0.750 & $\begin{array}{l}0.58(0.29- \\
1.17)\end{array}$ & 0.130 & $\begin{array}{l}0.87 \\
(0.74- \\
1.02)\end{array}$ & 0.089 \\
\hline 3 & $\begin{array}{l}1.00(0.82- \\
1.22)\end{array}$ & 0.998 & $\begin{array}{l}0.33(0.14- \\
0.82)\end{array}$ & $0.016 *$ & $\begin{array}{l}0.92 \\
(0.77- \\
1.10)\end{array}$ & 0.361 \\
\hline Academic & $\begin{array}{l}1.05(0.89- \\
1.25)\end{array}$ & 0.542 & $\begin{array}{l}1.03(0.50- \\
2.16)\end{array}$ & 0.927 & $\begin{array}{l}1.15 \\
(0.99- \\
1.34)\end{array}$ & 0.064 \\
\hline \multicolumn{7}{|c|}{$\mathrm{BI}=\mathrm{Barthel}$ Index; $\mathrm{BMI}=$ body mass index $; \mathrm{Cl}=$ confidence interval; $\mathrm{OR}=$ odds ratio; } \\
\hline$* p<0.05$ & & & & & & \\
\hline
\end{tabular}

Table 3 demonstrates the results of the multivariate logistic regression analyses for the worsening $\mathrm{BI}$ scores across all age groups. Age was a significant risk factor only in the pre-elderly and elderly groups. An mFl-5 score $\geq 2$ items was considered as a significant risk only in the non-elderly group (OR: $2.00 ; 95 \%$ Cl: $1.31-3.06)$, but not in the pre-elderly $(1.25 ; 0.85-1.84)$ and elderly $(0.97 ; 0.67-1.40)$ groups. The administration of oral anticoagulant medications on admission and several locations of meningiomas in the non-elderly and pre-elderly groups were also identified as significant risk factors. Figure 3 shows the results of the multivariate logistic regression analysis for the worsening BI scores across all age groups, depicted as forest plots for risk factors adjusted for other variables. 
Table 3

Multivariate logistic regression analyses for worsening BI scores across all age groups

\section{Objective variable}

Year age group (No.)

Sex male

Age (year)

\section{Worsening BI scores}

$\begin{array}{lll}\begin{array}{l}\text { Non-elderly; }<65 \\ (4,378)\end{array} & \text { Pre-elderly; 65-74 } & \text { Elderly; } \geq 75 \\ (2,421) & (1,339)\end{array}$

OR $(95 \% \mathrm{Cl}) \quad P-$ value $\mathrm{Cl})$

$P-$ value

OR $(95 \%$

$\mathrm{Cl})$

P.

value

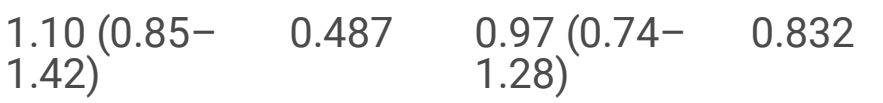

1.00

(0.76-

0.973

1.33)

$\begin{array}{lll}1.01(0.99- & 0.357 & 1.05(1.01- \\ 1.02) & 0.022^{*}\end{array}$

1.07

$<$

1.11)

0.001

*

BMI classification $(\mathrm{kg} / \mathrm{m} 2)$

$<18.5$

$1.00(0.64-$

1.57)

0.992

$1.21(0.76-$

0.427

1.25

(0.79-

0.350

1.97)

$18.5-24.9$

Reference

Reference

Reference

$25-29.9$

$1.30(0.98$

0.066

$0.82(0.60-\quad 0.200$

1.11)

0.95

(0.69-

0.732

1.72)

$\begin{array}{ll}0.559 & 0.89(0.49- \\ & 1.64)\end{array}$

1.30)

$30 \leq$

$0.86(0.5)$
$1.44)$

$1.64)$

0.718

0.69

(0.31-

0.348

1.51)

Medical history

Cerebral infarction

$1.53(0.56-\quad 0.404$

$1.83(0.89-$

0.099

1.33

(0.60-

0.479

4.17)

$3.75)$

2.96)

Angina pectoris

$0.68(0.20-\quad 0.532$

$0.76(0.35-\quad 0.476$

1.39

(0.79-

2.45)

The modified frailty index-5

\begin{tabular}{|c|c|c|c|c|c|c|}
\hline 0 item & Reference & & Reference & & Referen & \\
\hline 1 item & $\begin{array}{l}1.06(0.80- \\
1.41)\end{array}$ & 0.668 & $\begin{array}{l}1.20(0.91- \\
1.57)\end{array}$ & 0.192 & $\begin{array}{l}0.90 \\
(0.66- \\
1.22)\end{array}$ & 0.500 \\
\hline
\end{tabular}

$\mathrm{BI}=$ Barthel Index; $\mathrm{BMI}=$ body mass index $; \mathrm{Cl}=$ confidence interval; $\mathrm{OR}=$ odds ratio;

* $p<0.05$ 


\section{Objective variable}

$\geq 2$ items

Antiplatelet

Anticoagulation

Statin

Internal oral medication on

admission

1.86
3.59

$2.00(1.3$
$3.06)$

$1.25(0.85-$

1.84)
0.252

0.97

(0.67-

1.40)

0.863

$\begin{array}{lllllll} & 5.49) & & 5.69) & 0.001 * & (0.84- & \\ & & & & 2.14) & \\ \text { Statin } & 0.98(0.60- & 0.932 & 1.27(0.88- & 0.195 & 1.18 & 0.406 \\ & 1.60) & & 1.84) & & \begin{array}{l}1.80- \\ (1.74)\end{array} & \end{array}$

Location

Convexity

Reference

Reference

Reference

Falx

$2.21(1.48-$

3.30)

$1.43(0.94-\quad 0.093$

0.90

(0.55-

0.671

1.46)

Parasagittal

$2.55(1.80-$

0.001 * 2.16)

Parasagittal

3.62)

<.001*

$1.75(1.20-\quad 0.004$ *

1.29

(0.83-

0.257

2.01)

Lateral

$1.74(1.14-$
$2.65)$

0.010 *

$1.48(0.97-$
$2.25)$

0.068

0.97

(0.63-

0.900

1.50)

Midline

$1.37(0.87-$
$2.17)$

0.172

$1.30(0.84-$
$2.00)$

0.236

0.87

(0.48-

0.656

Posterior fossa

$1.34(0.89-$
$2.01)$

0.162

$1.18(0.80-\quad 0.408$

1.60)

Deep

1.76)

1.25
$(0.88-$
$1.81)$

$3.13(1.71-$

5.73)

0.001*

$2.85(1$
$6.34)$

1.51

(0.46-

0.496

4.99)

Hospital volume

1

Reference

Reference

Reference

$\mathrm{BI}=$ Barthel Index; $\mathrm{BMI}=$ body mass index $; \mathrm{Cl}=$ confidence interval; $\mathrm{OR}=$ odds ratio;

* $p<0.05$ 


\begin{tabular}{|c|c|c|c|c|c|c|}
\hline Objective variable & Worsening B & scores & & & & \\
\hline 2 & $\begin{array}{l}0.99(0.73- \\
1.36)\end{array}$ & 0.974 & $\begin{array}{l}0.98(0.72- \\
1.33)\end{array}$ & 0.881 & $\begin{array}{l}1.01 \\
(0.73- \\
1.38)\end{array}$ & 0.975 \\
\hline 3 & $\begin{array}{l}1.14(0.82- \\
1.61)\end{array}$ & 0.436 & $\begin{array}{l}0.86(0.61- \\
1.21)\end{array}$ & 0.372 & $\begin{array}{l}1.06 \\
(0.73- \\
1.54)\end{array}$ & 0.748 \\
\hline Academic & $\begin{array}{l}1.05(0.79- \\
1.39)\end{array}$ & 0.740 & $\begin{array}{l}1.29(0.96- \\
1.73)\end{array}$ & 0.086 & $\begin{array}{l}0.81 \\
(0.58- \\
1.12)\end{array}$ & 0.207 \\
\hline \multicolumn{7}{|c|}{$\mathrm{BI}=\mathrm{Barthel}$ Index; $\mathrm{BMI}=$ body mass index; $\mathrm{Cl}$ = confidence interval; $\mathrm{OR}$ = odds ratio; } \\
\hline \multicolumn{7}{|l|}{$* p<0.05$} \\
\hline
\end{tabular}

Table 4 shows the results of the multivariate logistic regression analyses for any complications across all age groups. Age was a significant risk factor only in the pre-elderly and elderly groups. An mFl-5 score of $\geq 2$ items was considered as a significant risk factor only in the non-elderly group (OR: $2.40 ; 95 \% \mathrm{Cl}$ :

$1.67-3.44)$, but not in the pre-elderly $(1.39 ; 1.00-1.94)$ and elderly $(1.19 ; 0.85-1.69)$ groups. Oral administration of anticoagulant and antiplatelet medications on admission, several locations of meningioma in all groups, and admission in an academic hospital were significant risk factors in the preelderly group. Figure 4 shows the results of the multivariate logistic regression analysis for any complications in all age groups, depicted in the form of forest plots for risk factors adjusted for other variables. 
Table 4

Multivariate logistic regression analyses for any complications across all age groups

\section{Objective variable}

\begin{tabular}{|c|c|c|c|c|c|c|}
\hline \multirow[t]{2}{*}{ Year age group (No.) } & \multicolumn{2}{|c|}{$\begin{array}{l}\text { Non-elderly; }<65 \\
(4,378)\end{array}$} & \multicolumn{2}{|c|}{$\begin{array}{l}\text { Pre-elderly; } 65-74 \\
(2,421)\end{array}$} & \multicolumn{2}{|c|}{ Elderly; $\geq 75(1,339)$} \\
\hline & OR $(95 \% \mathrm{Cl})$ & $\begin{array}{l}P \text { - } \\
\text { value }\end{array}$ & $\begin{array}{l}\mathrm{OR}(95 \% \\
\mathrm{Cl})\end{array}$ & $\begin{array}{l}P \text { - } \\
\text { value }\end{array}$ & $\begin{array}{l}\mathrm{OR}(95 \% \\
\mathrm{Cl})\end{array}$ & $\begin{array}{l}P \text { - } \\
\text { value }\end{array}$ \\
\hline Sex male & $\begin{array}{l}1.07(0.85- \\
1.33)\end{array}$ & 0.572 & $\begin{array}{l}1.08 \\
(0.85- \\
1.37)\end{array}$ & 0.509 & $\begin{array}{l}1.04 \\
(0.80- \\
1.36)\end{array}$ & 0.757 \\
\hline Age (year) & $\begin{array}{l}1.00(0.99- \\
1.01)\end{array}$ & 0.861 & $\begin{array}{l}1.04(1.00- \\
1.08)\end{array}$ & $0.034 *$ & $\begin{array}{l}1.08 \\
(1.04- \\
1.12)\end{array}$ & $\begin{array}{l}< \\
0.001 \\
\star\end{array}$ \\
\hline \multicolumn{7}{|c|}{ BMI classification $(\mathrm{kg} / \mathrm{m} 2)$} \\
\hline$<18.5$ & $\begin{array}{l}1.23(0.86- \\
1.75)\end{array}$ & 0.250 & $\begin{array}{l}1.17 \\
(0.77- \\
1.77)\end{array}$ & 0.471 & $\begin{array}{l}1.35 \\
(0.87- \\
2.08)\end{array}$ & 0.181 \\
\hline $18.5-24.9$ & Reference & & Reference & & Reference & \\
\hline $25-29.9$ & $\begin{array}{l}1.21(0.95- \\
1.54)\end{array}$ & 0.121 & $\begin{array}{l}0.88 \\
(0.68- \\
1.16)\end{array}$ & 0.382 & $\begin{array}{l}1.01 \\
(0.75- \\
1.36)\end{array}$ & 0.957 \\
\hline $30 \leq$ & $\begin{array}{l}0.83(0.54- \\
1.30)\end{array}$ & 0.419 & $\begin{array}{l}0.97 \\
(0.57- \\
1.64)\end{array}$ & 0.909 & $\begin{array}{l}0.87 \\
(0.44- \\
1.73)\end{array}$ & 0.701 \\
\hline \multicolumn{7}{|l|}{ Medical history } \\
\hline Cerebral infarction & $\begin{array}{l}0.83(0.31- \\
2.27)\end{array}$ & 0.721 & $\begin{array}{l}1.51 \\
(0.78- \\
2.93)\end{array}$ & 0.221 & $\begin{array}{l}1.24 \\
(0.58- \\
2.65)\end{array}$ & 0.578 \\
\hline Angina pectoris & $\begin{array}{l}0.59(0.20- \\
1.73)\end{array}$ & 0.337 & $\begin{array}{l}0.62 \\
(0.31- \\
1.23)\end{array}$ & 0.175 & $\begin{array}{l}1.14 \\
(0.66- \\
1.98)\end{array}$ & 0.643 \\
\hline \multicolumn{7}{|c|}{$\begin{array}{l}\text { The modified frailty index- } \\
5\end{array}$} \\
\hline 0 item & Reference & & Reference & & Reference & \\
\hline 1 item & $\begin{array}{l}1.10(0.87- \\
1.40)\end{array}$ & 0.425 & $\begin{array}{l}1.22 \\
(0.97- \\
1.55)\end{array}$ & 0.094 & $\begin{array}{l}1.01 \\
(0.76- \\
1.35)\end{array}$ & 0.941 \\
\hline
\end{tabular}

$\mathrm{BI}=$ Barthel Index; $\mathrm{BMI}=$ body mass index $; \mathrm{Cl}=$ confidence interval; $\mathrm{OR}=$ odds ratio;

$* p<0.05$ 


\section{Objective variable}

$\geq 2$ items

ternal oral medication on

admission

Antiplatelet

Anticoagulation

Statin

Location
Convexity

Falx

Parasagittal

Lateral

Midline

Posterior fossa

Posterior fossa

Deep

$2.22(1.28-$
$3.84)$

$1.13(0.7$
$1.65)$

$2.07(1.55-$

2.78)

$3.61(2.14-$
$6.08)$

$\begin{array}{ll}<.001 * & 2.01 \\ & (1.33- \\ & 3.04)\end{array}$

$3.08(1.78-$
$5.33)$

$<.001$ *

3.31

(2.18-

5.01)

$\begin{array}{ll}0.68(0.43- & 0.098 \\ 1.07)\end{array}$

1.18

(0.86-

1.64)

\section{Any complications}

1.19

(0.85-

1.69)

0.303

$.39(1.00-\quad 0.050$

(1)

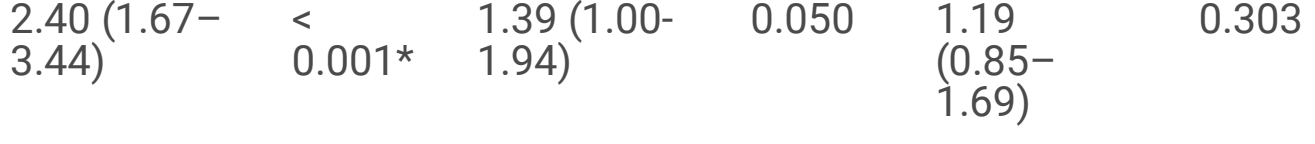




\begin{tabular}{|c|c|c|c|c|c|c|}
\hline Objective variable & Any complic & ions & & & & \\
\hline 2 & $\begin{array}{l}0.76(0.58- \\
1.00)\end{array}$ & 0.051 & $\begin{array}{l}0.99 \\
(0.76- \\
1.30)\end{array}$ & 0.964 & $\begin{array}{l}0.93 \\
(0.69- \\
1.26)\end{array}$ & 0.652 \\
\hline 3 & $\begin{array}{l}1.00(0.76- \\
1.33)\end{array}$ & 0.975 & $\begin{array}{l}0.83 \\
(0.61- \\
1.12)\end{array}$ & 0.219 & $\begin{array}{l}0.93 \\
(0.65- \\
1.32)\end{array}$ & 0.666 \\
\hline Academic & $\begin{array}{l}1.20(0.95- \\
1.53)\end{array}$ & 0.130 & $\begin{array}{l}1.34 \\
(1.04- \\
1.74)\end{array}$ & $0.024^{\star}$ & $\begin{array}{l}0.83 \\
(0.60- \\
1.13)\end{array}$ & 0.229 \\
\hline \multicolumn{7}{|c|}{$\mathrm{BI}=\mathrm{Barthel}$ Index; $\mathrm{BMI}=$ body mass index; $\mathrm{Cl}=$ confidence interval; $\mathrm{OR}=$ odds ratio; } \\
\hline \multicolumn{7}{|l|}{$* p<0.05$} \\
\hline
\end{tabular}

\section{Discussion}

In this study, worsening $\mathrm{BI}$ and $\mathrm{mFI}-5$ scores and the proportion of patients with medical history were significantly increased with age. An mFI- 5 score $\geq 2$ items was a significant risk factor for worsening $\mathrm{BI}$ scores, in-hospital mortality, and any complications after surgery. In the analysis of all age groups, an $\mathrm{mFI}-5$ score $\geq 2$ items was a significant risk factor for worsening $\mathrm{BI}$ scores and any complications after surgery only in the non-elderly group, but not in the pre-elderly and elderly groups.

\section{The rate of mortality and complications after surgery}

The rates of in-hospital mortality and any complications after surgery in our study were $0.5-0.9 \%$ and $10.4-25.5 \%$, respectively. These rates were similar to those observed in previous studies; the rates of mortality and complications after surgery were reported as $0.5-1.5 \%$, and $6.8-14.8 \%$ respectively $[5,8$, 15 , 27]. A recent systematic review reported that in elderly patients with meningiomas, the rate of one-year postoperative mortality and neurological complications after surgery ranged from $2.7-49.4 \%$ and from $0-16.7 \%$, respectively [9].

\section{$\mathrm{mFI}$ scores and age as predictors of surgical complications and worsening}

In both sexes, there was a significant non-linear association between age and the FI scores.[22] Overall, the FI scores were much stronger predictors of mortality than age [22]. In this study, the mFI-5 scores increased with age, similar to findings from a previous report investigating the FI [12]. Aging is a heterogeneous process and chronologic age is not necessarily synonymous with an individual's health status. Even so, there is little evidence to determine whether there are differences in the frailty criteria in younger versus older adults. The relative risk of mortality in younger adults is associated more with frailty than age [21]. In the present study, the overall background of $\mathrm{mFI}-5$ scores in the older groups were relatively higher; therefore, the $\mathrm{mFI-5}$ may not be a significant predictor in advanced age groups. The $\mathrm{mFI}$ 
5 is a useful predictor of complications and worsening after surgery, specifically in non-elderly rather than elderly patients with meningiomas. While this may sound paradoxical at first, we believe that it may be true. In meningioma surgeries, care must be taken when making decisions using the mFI- 5 scores based on the patients' age. Furthermore, we can recommend preoperative fitness recovery to improve frailty, especially in non-elderly patients, who have a greater potential to regain fitness than the elderly [21].

\section{Advanced age as a risk factor of surgical complication and worsening Bl score}

We assessed the worsening $\mathrm{BI}$ scores between admission and discharge and found that the rate of worsening $\mathrm{BI}$ scores increased from 7.1-21.1\% (mean $=11.0 \%$ ) as age increased, which is comparable to the increase reported in previous studies $(8.3-14.8 \%) .[3,5,27]$ We found pneumonia and the worsening $\mathrm{BI}$ scores to be significantly correlated with advanced age in our study. The most common complications after surgery for meningioma were new focal neurological deficits and pneumonia [5, 27]. Notably, these two complications are currently considered common and inevitable in advanced aged patients with meningiomas [27].

However, we found no significant association between advanced age and in-hospital mortality. According to a recent systematic review of elderly patients with meningioma surgery, the rate of in-hospital mortality, worsening of the postoperative performance status, neurological deficits, and general complications ranged widely based on the reports and were not necessarily associated with advanced-age [9].

\section{Other risk factors in meningioma surgery}

The administration of antiplatelet and anticoagulation drugs was found to be a risk factor for worse outcomes in previous neurosurgical studies [1]. In our study, these medications were also risk factors for worsening Bl scores, in-hospital mortality, and any complications after surgery regardless of the patients' age. Antithrombotic drugs have been administered for the prevention of recurrence in cardiac and cerebral vascular events, necessitating the correct use of these drugs in the elderly. Minimum necessary use of antithrombotic medication is required, especially in the elderly during the perioperative period since antithrombotic drugs are more frequently administered to the elderly.

Several risk factors for mortality in meningioma surgery in the elderly have been identified, such as location [20], preoperative Karnofsky performance status [6], the BI score [4], other grading systems [24] and advanced age [19]. Although parasagittal and deep locations of the meningioma were significant risk factors for the worsening BI scores and any complications in both non-elderly and pre-elderly groups, they were no longer risk factors in the elderly group (Tables 3 and 4, Figs. 3 and 4). This phenomenon may be due to differences in aggressive treatment based on the location, delicate treatment management in the elderly, and the selective indication of surgery in elderly patients with meningiomas.

In this study, the in-hospital mortality in high volume hospitals was a significant inverse risk factor, due to the ability of high volume hospitals to rescue patients from major perioperative complications [16]. Academic hospitals were found to be significant risk factors for postoperative complications in pre- 
elderly patients. This may be likely because academic hospitals tend to receive the most serious and difficult cases.

\section{Limitations}

This study has some limitations. First, the DPC database does not include post-discharge data, meningioma size, pathological findings, and the extent of resection and perioperative radiation therapy. Therefore, we could not assess the long-term status of recurrence or BI scores after discharge. These variables are usually not recorded in other administrative in-hospital databases. Second, this was a registry-based study, not a randomized controlled study. Therefore, we could not completely exclude bias. However, we improved the integrity of this study by analyzing the location of meningiomas, as well as preoperative and postoperative BI scores. Third, some variables had wide Cls due to a limited number of cases. Fourth, our results may not be generalizable to other countries, which have different medical resources and systems, and they must be interpreted with caution considering that Japan has the highest proportion of elderly people worldwide.

\section{Conclusions}

Although advanced age could lead to postoperative functional decline and complications at discharge, the $\mathrm{mFI}-5$ scores could predict the complication and worsening in the patients aged $<65$ years, but not in the patients aged $\geq 65$ years. Care must be taken when making decisions using the $\mathrm{mFI}-5$ scores based on the patients' age.

\section{Declarations}

\section{Funding:}

This work was supported by the Japan Society for the Promotion of Science, Grant-in-Aid for Scientific Research (C) 17K10829.

\section{Role of the Funding Source:}

The funders of the study had no role in study design, data collection, data analysis, data interpretation, or writing of this report. The corresponding author has full access to all data in the study and has final responsibility for the decision to submit for publication.

\section{Conflict of interest:}

All authors report no conflict of interest concerning the materials or methods used in this study or the findings specified in this paper.

\section{Data availability}


The anonymized data for this study could be shared on the request of any qualified investigator to the corresponding author. Only the results of primary data from the DPC could be made available for reasonable requests in accordance with the review board.

\section{Code availability}

Stata software (version 15; StataCorp, College Station, TX, USA)

\section{Contributors:}

All authors made contributions to the intellectual content of this paper, contributed to data interpretation, approved the final manuscript, and agreed with submission to this journal. Fusao Ikawa: study design and concept; acquired funding; conducted research; collected, curated, managed, and analyzed data; quality control; statistical analysis; and drafted the manuscript. Nobuaki Michihata: collected, curated, and analyzed data; quality control; statistical analysis; and revised the manuscript. Soichi Oya, Toshikazu Hidaka, Shingo Matsuda, Iori Ozono, Kenji Ohata, Kiyoshi Saito, and Kazunari Yoshida: conceived and oversaw the study, as well as revised the manuscript. Kiyohide Fushimi, Hideo Yasunaga, Teiji Tominaga, and Kaoru Kurisu: conceived and oversaw the study, as well as assisted in collecting data.

\section{Ethics approval}

The present study was approved by the local Institutional Review Boards (no. E-631 and no. 3501-[1]). Due to the anonymous nature of the data in this study, the requirement for informed consent was waived.

\section{Consent to participate}

Not applicable

\section{Consent for publication}

Not applicable

\section{Acknowledgments:}

The authors sincerely thank all persons from participating institutions in the Diagnosis Procedure Combination database. The authors thank Ms. Tomoko Saegusa, for her assistance.

\section{References}

1. Algra AM, Lindgren A, Vergouwen MDI, Greving JP, van der Schaaf IC, van Doormaal TPC, Rinkel GJE (2019) Procedural clinical complications, case-fatality risks, and risk factors in endovascular and neurosurgical treatment of unruptured intracranial aneurysms: A systematic review and metaanalysis JAMA Neurol 76:282-293. https://doi.org/10.1001/jamaneurol.2018.4165 
2. Bilgin S, Kose N, Karakaya J, Mut M (2014) Traumatic brain injury shows better functional recovery than brain tumor: a rehabilitative perspective. Eur J Phys Rehabil Med 50:17-23.

3. Chan RC, Thompson GB (1984) Morbidity, mortality, and quality of life following surgery for intracranial meningiomas. A retrospective study in 257 cases. J Neurosurg 60:52-60. https://doi.org/10.3171/jns.1984.60.1.0052

4. Cohen-Inbar O, Soustiel JF, Zaaroor M (2010) Meningiomas in the elderly, the surgical benefit and a new scoring system. Acta Neurochir (Wien) 152:87-97. https://doi.org/10.1007/s00701-009-0552-6

5. Corell A, Thurin E, Skoglund T, Farahmand D, Henriksson R, Rydenhag B, Gulati S, Bartek J, Jr., Jakola AS (2019) Neurosurgical treatment and outcome patterns of meningioma in Sweden: a nationwide registry-based study. Acta Neurochir (Wien) 161:333-341. https://doi.org/10.1007/s00701-01903799-3

6. D'Andrea G, Roperto R, Caroli E, Crispo F, Ferrante L (2005) Thirty-seven cases of intracranial meningiomas in the ninth decade of life: Our experience and review of the literature. Neurosurgery 56:956-961.

7. Farhat JS, Velanovich V, Falvo AJ, Horst HM, Swartz A, Patton JH, Jr., Rubinfeld IS (2012) Are the frail destined to fail? Frailty index as predictor of surgical morbidity and mortality in the elderly. J Trauma Acute Care Surg 72:1526-1531. https://doi.org/10.1097/TA.0b013e3182542fab

8. Gerlach R, Raabe A, Scharrer I, Meixensberger J, Seifert V (2004) Post-operative hematoma after surgery for intracranial meningiomas: causes, avoidable risk factors and clinical outcome. Neurol Res 26:61-66. https://doi.org/10.1179/016164104773026543

9. Ikawa F, Kinoshita Y, Takeda M, Saito T, Yamaguchi S, Yamasaki F, lida K, Sugiyama K, Arita K, Kurisu K (2017) Review of current evidence regarding surgery in elderly patients with meningioma. Neurol Med Chir (Tokyo) 57:521-533. https://doi.org/10.2176/nmc.ra.2017-0011

10. Ikawa F, Michihata N, Akiyama Y, lihara K, Matano F, Morita A, Kato Y, lida K, Kurisu K, Fushimi K, Yasunaga $\mathrm{H}$ (2019) Treatment risk for elderly patients with unruptured cerebral aneurysm from a nationwide database in Japan. World Neurosurg 132:e89-e98.

https://doi.org/10.1016/j.wneu.2019.08.252

11. Ikawa F, Michihata N, Matsushige T, Abiko M, Ishii D, Oshita J, Okazaki T, Sakamoto S, Kurogi R, lihara K, Nishimura K, Morita A, Fushimi K, Yasunaga H, Kurisu K (2020) In-hospital mortality and poor outcome after surgical clipping and endovascular coiling for aneurysmal subarachnoid hemorrhage using nationwide databases: a systematic review and meta-analysis. Neurosurg Rev 43:655-667. https://doi.org/10.1007/s10143-019-01096-2

12. Kehler DS, Ferguson T, Stammers AN, Bohm C, Arora RC, Duhamel TA, Tangri N (2017) Prevalence of frailty in Canadians 18-79 years old in the Canadian Health Measures Survey. BMC Geriatrics 17. https://doi.org/10.1186/s12877-017-0423-6

13. Khalafallah AM, Huq S, Jimenez AE, Brem H, Mukherjee D (2020) The 5-factor modified frailty index: an effective predictor of mortality in brain tumor patients. J Neurosurg Published online August 14, 2020:1-9. https://doi.org/10.3171/2020.5.JNS20766 
14. Kuratsu J, Kochi M, Ushio Y (2000) Incidence and clinical features of asymptomatic meningiomas. J Neurosurg 92:766-770. https://doi.org/10.3171/jns.2000.92.5.0766

15. Lassen B, Helseth E, Ronning P, Scheie D, Johannesen TB, Maehlen J, Langmoen IA, Meling TR (2011) Surgical mortality at 30 days and complications leading to recraniotomy in 2630 consecutive craniotomies for intracranial tumors. Neurosurgery 68:1259-1269.

https://doi.org/10.1227/NEU.0b013e31820c0441

16. Malone H, Cloney M, Yang J, Hershman DL, Wright JD, Neugut Al, Bruce JN (2018) Failure to Rescue and Mortality Following Resection of Intracranial Neoplasms. Neurosurgery 83:263-269. https://doi.org/10.1093/neuros/nyx354

17. Morita K, Matsui H, Michihata N, Fushimi K, Yasunaga H (2019) Association of early systemic corticosteroid therapy with mortality in patients with Stevens-Johnson syndrome or toxic epidermal necrolysis: a retrospective cohort study using a nationwide claims database. Am J Clin Dermatol 20:579-592. https://doi.org/10.1007/s40257-019-00443-9

18. Orimo H, Ito H, Suzuki T, Araki A, Hosoi T, Sawabe M (2006) Reviewing the definition of "elderly". Geriatr Gerontol Int 6:149-158. https://doi.org/10.1111/j.1447-0594.2006.00341.x

19. Patil CG, Veeravagu A, Lad SP, Boakye M (2010) Craniotomy for resection of meningioma in the elderly: A multicentre, prospective analysis from the National Surgical Quality Improvement Program. J Neurol Neurosurg Psychiatry 81:502-505. https://doi.org/10.1136/jnnp.2009.185074

20. Reinert M, Babey M, Curschmann J, Vajtai I, Seiler RW, Mariani L (2006) Morbidity in 201 patients with small sized meningioma treated by microsurgery. Acta Neurochir (Wien) 148:1257-1266. https://doi.org/10.1007/s00701-006-0909-z

21. Rockwood K, Song X, Mitnitski A (2011) Changes in relative fitness and frailty across the adult lifespan: evidence from the Canadian National Population Health Survey. Cmaj 183:E487-494. https://doi.org/10.1503/cmaj.101271

22. Romero-Ortuno R, Kenny RA (2012) The frailty index in Europeans: association with age and mortality. Age and ageing 41:684-689. https://doi.org/10.1093/ageing/afs051

23. Rubinfeld I, Farooq M, Velanovich V, Syed Z (2010) Predicting Surgical Risk: How Much Data is Enough? AMIA Annu Symp Proc 2010:777-781.

24. Sacko O, Sesay M, Roux FE, Riem T, Grenier B, Liguoro D, Loiseau H (2007) Intracranial meningioma surgery in the ninth decade of life. Neurosurgery 61:950-955.

https://doi.org/10.1227/01.neu.0000303190.80049.7d

25. Saxton A, Velanovich V (2011) Preoperative frailty and quality of life as predictors of postoperative complications. Ann Surg 253:1223-1229. https://doi.org/10.1097/SLA.0b013e318214bce7

26. Subramaniam S, Aalberg JJ, Soriano RP, Divino CM (2018) New 5-Factor Modified Frailty Index Using American College of Surgeons NSQIP Data. J Am Coll Surg 226:173-182. https://doi.org/10.1016/j.jamcollsurg.2017.11.005

27. Sughrue ME, Rutkowski MJ, Shangari G, Chang HQ, Parsa AT, Berger MS, McDermott MW (2011) Risk factors for the development of serious medical complications after resection of meningiomas. 
Clinical article. J Neurosurg 114:697-704. https://doi.org/10.3171/2010.6.Jns091974

28. Traven SA, Reeves RA, Sekar MG, Slone HS, Walton ZJ (2019) New 5-Factor Modified Frailty Index Predicts Morbidity and Mortality in Primary Hip and Knee Arthroplasty. J Arthroplasty 34:140-144. https://doi.org/10.1016/j.arth.2018.09.040

29. Van der Putten JJ, Hobart JC, Freeman JA, Thompson AJ (1999) Measuring change in disability after inpatient rehabilitation: comparison of the responsiveness of the Barthel index and the Functional Independence Measure. J Neurol Neurosurg Psychiatry 66:480-484. https://doi.org/10.1136/jnnp.66.4.480

30. Wahl TS, Graham LA, Hawn MT, Richman J, Hollis RH, Jones CE, Copeland LA, Burns EA, Itani KM, Morris MS (2017) Association of the Modified Frailty Index With 30-Day Surgical Readmission. JAMA Surg 152:749-757. https://doi.org/10.1001/jamasurg.2017.1025

31. Weaver DJ, Malik AT, Jain N, Yu E, Kim J, Khan SN (2019) The Modified 5-Item Frailty Index: A Concise and Useful Tool for Assessing the Impact of Frailty on Postoperative Morbidity Following Elective Posterior Lumbar Fusions. World Neurosurg 124:e626-e632. https://doi.org/10.1016/j.wneu.2018.12.168

32. Yamana H, Moriwaki M, Horiguchi H, Kodan M, Fushimi K, Yasunaga H (2017) Validity of diagnoses, procedures, and laboratory data in Japanese administrative data. J Epidemiol 27:476-482. https://doi.org/10.1016/j.je.2016.09.009

33. Youngerman BE, Neugut Al, Yang J, Hershman DL, Wright JD, Bruce JN (2018) The modified frailty index and 30-day adverse events in oncologic neurosurgery. J Neurooncol 136:197-206. https://doi.org/10.1007/s11060-017-2644-0

\section{Figures}




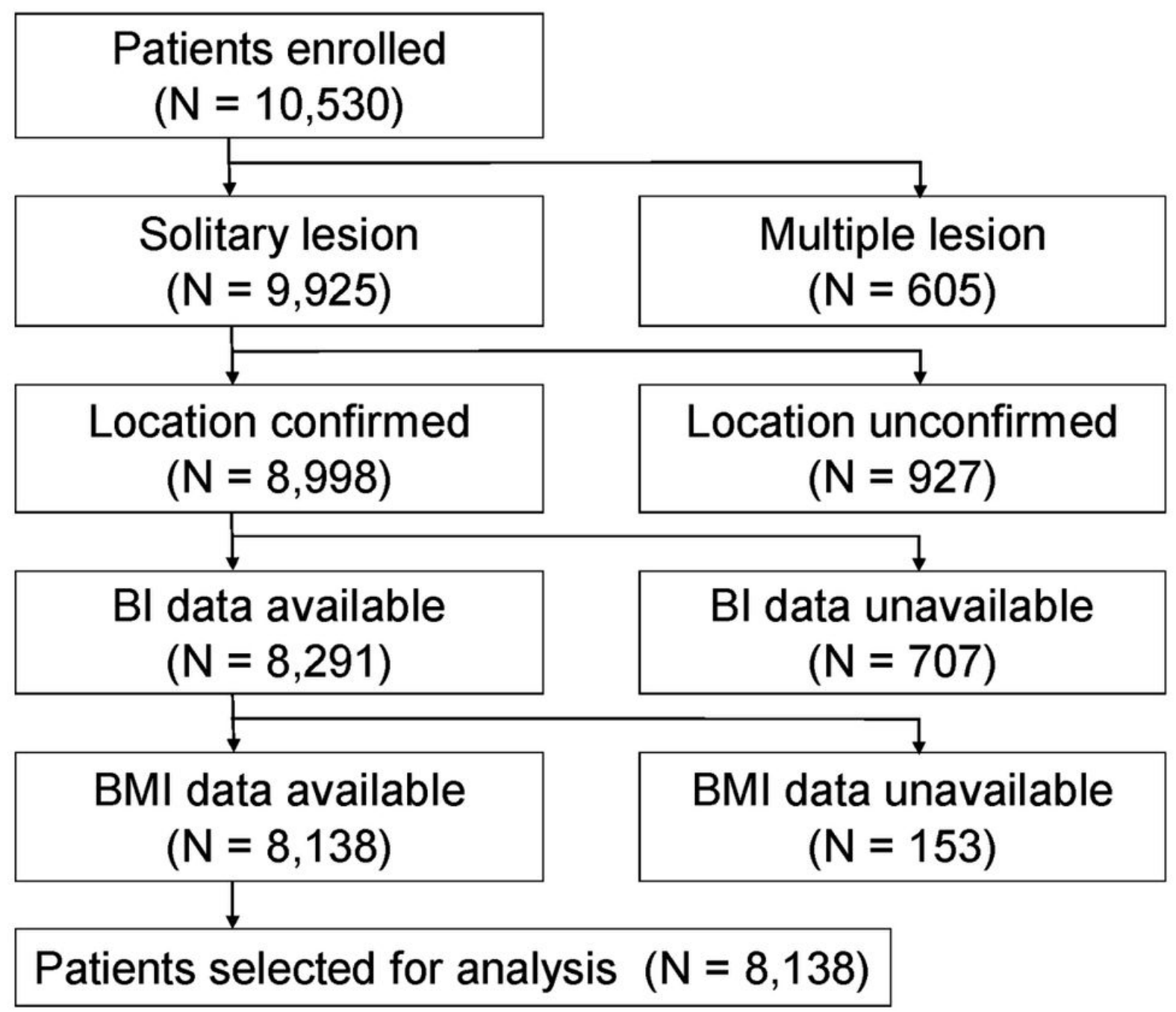

Figure 1

Selection of patients with surgically treated meningiomas Abbreviations: BI, Barthel Index; BMI, body mass index 

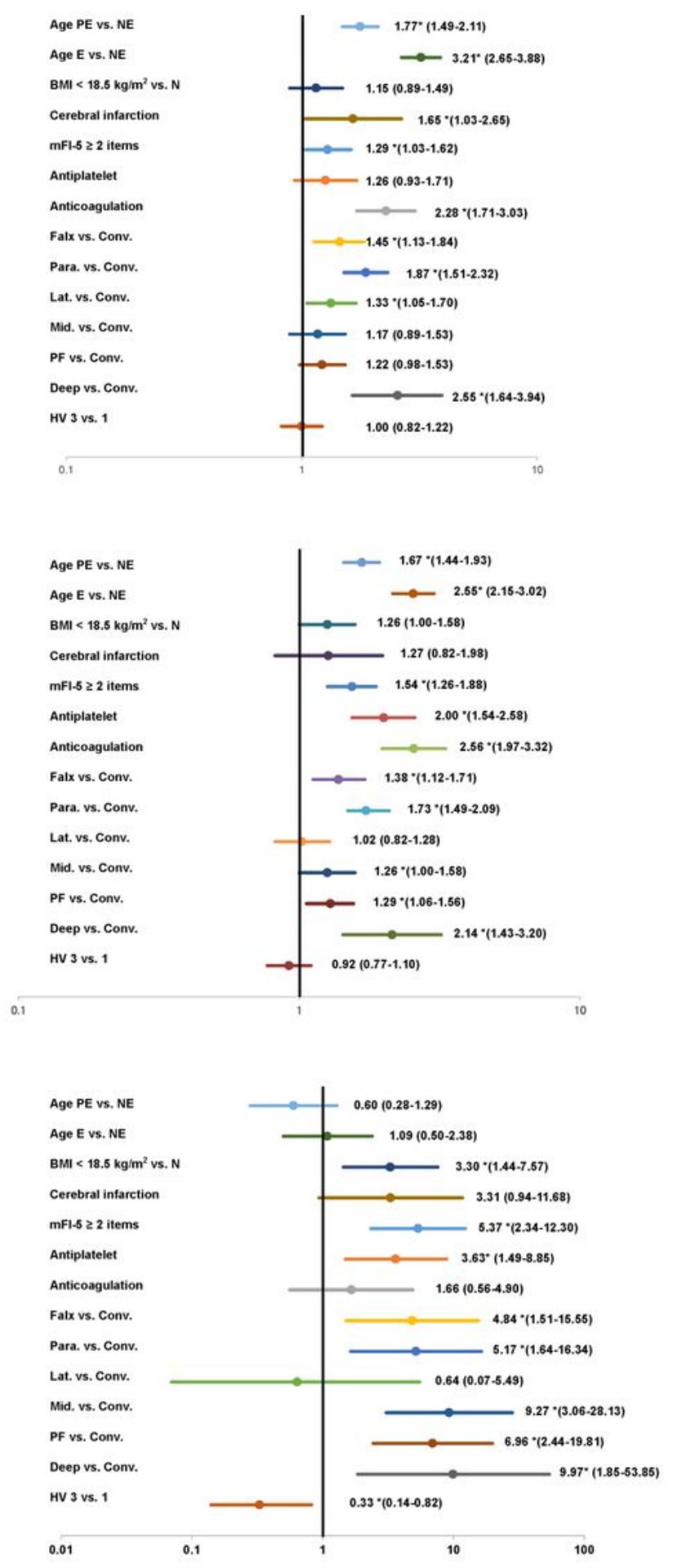

\section{Figure 2}

Forest plots for risk factors adjusted for other variables. (a) The worsening BI scores. (b) In-hospital mortality. (c) Any complications Abbreviations: BI, Barthel Index; BMI, body mass index; Conv, convexity; E, elderly; HV, hospital volume; Lat, lateral; Mid, midline; N, normal; Para, parasagittal; PE, pre-elderly; PF, posterior fossa. ${ }^{*} \mathrm{p}<0.05$ 


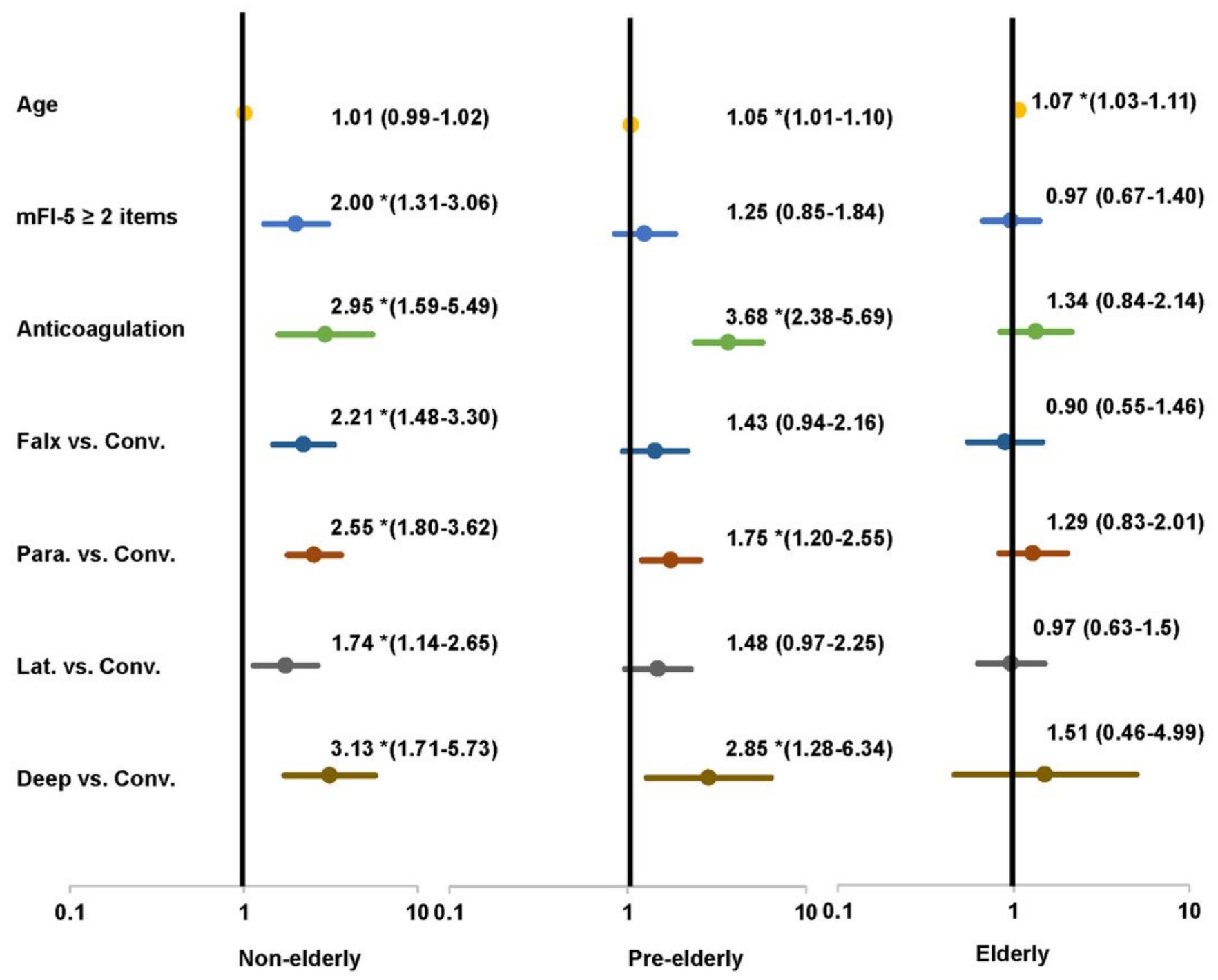

Figure 3

Forest plots of the risk factors adjusted for other variables for the worsening $\mathrm{BI}$ scores in the non-elderly, pre-elderly, and elderly Abbreviations: Conv, convexity; E, Lat, lateral; Para, parasagittal; PF, posterior fossa. ${ }^{*} p<0.05$ 


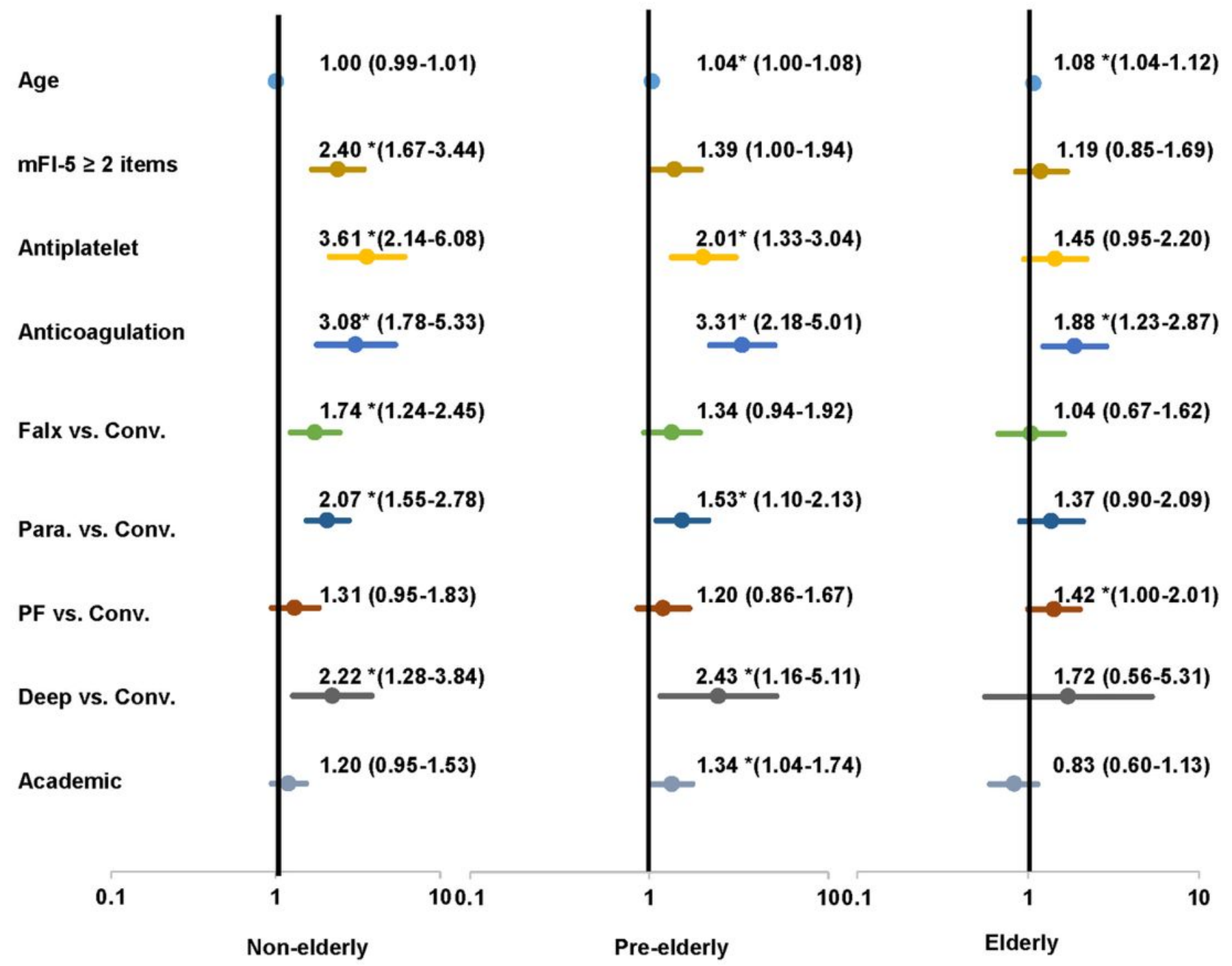

Figure 4

Forest plots of the risk factors adjusted for other variables for any complications in the non-elderly, preelderly, and elderly Abbreviations: Conv, convexity; Para, parasagittal; PF, posterior fossa. ${ }^{*} p<0.05$ 